PAPER

\section{X-ray grating interferometry design for the 4D GRAPH-X system}

To cite this article: Alessandra Patera et al 2022 J. Phys. D: Appl. Phys. 55045103

View the article online for updates and enhancements.
You may also like

- Mathematical Optimization of the Spatial
$\frac{\text { Distribution of Platinum Particles in the }}{\text { Catalyst Layer of Pemfcs }}$
James Lamb, Grayson Mixon and Petru
Andrei
- Practical implementation of tetrahedral
$\frac{\text { mesh reconstruction in emission }}{\text { tomography }}$
R Boutchko, A Sitek and G T Gullberg
- Large-Scale Optimization of Polymer
Electrolyte Membrane Fuel Cells
Petru Andrei and Mohit Mehta




\title{
$X$-ray grating interferometry design for the 4D GRAPH-X system
}

\author{
Alessandra Patera $^{1, *}$, Carolina Arboleda $^{2}$, Veronica Ferrero $^{1}$, Elisa Fiorina ${ }^{1}$, \\ Konstantins Jefimovs ${ }^{2}$, Alessandro Lo Giudice ${ }^{1,3}$, Felix Mas Milian ${ }^{3}$, Paolo Mereu ${ }^{1}$,

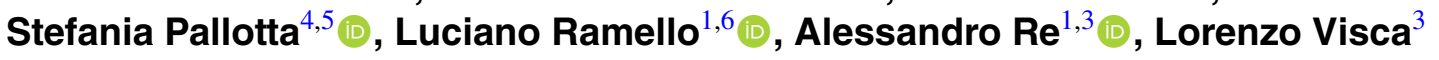 \\ and Piergiorgio Cerello ${ }^{1}$
}

${ }^{1}$ Sezione di Torino, National Institute for Nuclear Physics (INFN), Torino, Italy

${ }^{2}$ Swiss Light Source, Paul Scherrer Institute, Villigen, Switzerland

${ }^{3}$ Physics Department, University of Torino, Torino, Italy

${ }^{4}$ Department of Experimental and Clinical Biomedical Sciences, University of Florence, Florence, Italy

${ }^{5}$ Medical Physics Unit, Azienda Ospedaliero Universitaria Careggi, Florence, Italy

${ }^{6}$ Dipartimento di Scienze e Innovazione Tecnologica, Università del Piemonte Orientale, Alessandria, Italy

E-mail: patera.alessandra85@gmail.com

Received 7 October 2020, revised 14 September 2021

Accepted for publication 14 October 2021

Published 25 October 2021

\begin{abstract}
The 4D GRAPH-X (Dynamic GRAting-based PHase contrast X-ray imaging) project aims at developing a prototype of an X-ray grating-based phase-contrast imaging scanner in a laboratory setting, which is based on the Moirè single-shot acquisition method in order to be optimized for analysing moving objects (in the specific case, a dynamic thorax phantom), that could evolve into a suitable tool for biomedical applications although it can be extended to other application fields. When designing an x-ray Talbot-Lau interferometer, high visibility and sensitivity are two important figures of merit, strictly related to the performance of the system in obtaining high quality phase contrast and dark-field images. Wave field simulations are performed to optimize the setup specifications and construct a high-resolution and high-sensitivity imaging system. In this work, the design of a dynamic imaging setup using a conventional milli-focus x-ray source is presented. Optimization by wave front simulations leads to a symmetric configuration with $5.25 \mu \mathrm{m}$ pitch at third Talbot order and $45 \mathrm{keV}$ design energy. The simulated visibility is about $22 \%$. Results from GATE based Monte Carlo simulations show a 19\% transmission percentage of the incoming beam into the detector after passing through all the gratings and the sample. Such results are promising in view of building a system optimized for dynamic imaging.
\end{abstract}

Keywords: $\mathrm{x}$-ray phase contrast imaging, talbot-lau grating interferometer, dynamic imaging, wavefront simulation

(Some figures may appear in colour only in the online journal)

\section{Introduction}

Exploiting x-rays as an imaging modality requires a deep understanding of their interaction with matter. The imaging principle is based on analyzing $\mathrm{X}$-rays that have passed through

* Author to whom any correspondence should be addressed. the object of interest and using this information to derive its material properties. The different kinds of interaction mechanisms of $\mathrm{x}$-rays with matter yield specific and complementary information about the material properties of the object. In wave optics, the interaction of $x$-rays with matter is described by the refraction index $(n=1-\delta+i \beta)$, whose imaginary $(\beta)$ and real $(\delta)$ parts are related to the attenuation and the phase shift of x-rays, respectively. It is important to point out that 
the $\delta / \beta$ ratio quadratically increases with the energy thus enhancing the relative importance of phase-sensitive imaging when the energy increases.

Phase-sensitive x-ray imaging has been attracting attention since the late 1990s, with the development of digital $\mathrm{x}$-ray imaging technology and synchrotron radiation facilities [1]. Conventional x-ray imaging maps the attenuation coefficient-and therefore the electronic density-of materials, which mainly depends on the atomic number (Z) [2] and is described by the imaginary part of the refractive index. Therefore, it is poorly sensitive to weakly absorbing materials, such as soft tissue. Conversely, $x$-ray phase contrast imaging, which is linked to the real part of the complex refractive index [3], allows the visualization of soft materials by relying on the phase shift or the refraction of x-rays caused by the object. Indeed, in the 40-100 keV energy range, phase contrast $\mathrm{x}$ ray imaging shows an increase of the signal-to-noise ratio (SNR) by up to two orders of magnitude over conventional (i.e. absorption-based) imaging [4]. Among a variety of phasesensitive techniques [5-11], x-ray grating interferometry (GI) has been successfully implemented both in laboratory systems based on conventional low-brilliance x-ray tubes (Talbot-Lau type) [11] and at synchrotrons (Talbot type) [12, 13]. This method provides information about the attenuation, phaseshift and scattering properties of the sample, generating three independent images with a simultaneous acquisition. The absorption image (ABS) yields information about the spatial distribution of the linear attenuation coefficient, while the differential phase contrast projections (DPC) are related to the refractive index decrement and the dark field signal (DF) allows the reconstruction of the linear diffusion coefficient [14]. In particular, the DF signal is determined by small-angle $\mathrm{x}$-ray scattering on structures typically below the spatial resolution of a conventional imaging setup [15]. Thus, x-ray GI not only yields complementary information within the same acquisition session, but also allows obtaining information that, because of the resolution limit, is not accessible for conventional $\mathrm{x}$-ray imaging, providing a better sensitivity on low-density or small structures, a crucial feature in view of biomedical applications [16-18]. Lung imaging is one of the most promising applications for x-ray dark-field imaging because, in the presence of lung diseases, the alveoli structure changes [19], thus generating a decrease in the dark-field signal, which is more sensitive to slight changes than the attenuation signal. Several publications describe ex-vivo and in-vivo investigations on the lungs [20-22], while the potential of x-ray dark-field imaging of in-situ human lungs in a deceased body was recently discussed [19].

The 4D GRAPH-X project aims at implementing a prototype of a dynamic x-ray Talbot-Lau imaging system suitable for a range of applications in medicine with major focus on lung imaging. The purpose is to introduce a new lab-based $\mathrm{x}$-ray imaging acquisition and reconstruction protocol based on Moiré acquisition and analysis under dynamic conditions with reference to dynamic phantoms or moving objects. We selected a symmetric configuration of a laboratory-based $\mathrm{x}$-ray GI composed by a conventional milli-focus $\mathrm{x}$-ray source, a flat panel detector, a rotational stage to accommodate a small dynamic anthropomorphic thorax phantom and a system of three gratings with $5.25 \mu \mathrm{m}$ pitch. In the following section, an overview of the working principal is given prior introducing the specific system configuration. The optimization, design and constraints are discussed in detail. Finally, results on setting specifications, performance and validation are provided in section 3 followed by discussion and conclusion, in which the novelty of the following study is better explained.

\section{Materials and Methods}

\subsection{System working principle}

The first step in the design of a Talbot-Lau (TL) x-ray interferometer is the selection of the design energy, which must be compatible not only with the thickness and density of the objects to be examined, but also with the performance of the $\mathrm{x}$-ray tube and with the fabrication requirements of the gratings. Then, the interferometer is designed and optimized for the given source and detector system.

The TL interferometer requires a source grating G0, a phase grating G1, and an analyzer grating G2, as shown in figure 1 .

G0 is an absorption grating made of gold lines positioned directly after the source: it splits the source into an array of micron-sized sources, which are individually coherent, but mutually incoherent. G1, named phase grating, made of slightly absorbing lines, acts as a beam splitter: it divides the incident beam into different diffraction orders. The diffraction angle is very small, in the order of a few $\mu \mathrm{rad}$, leading to inline interference patterns downstream that appear as periodic intensity fringes. A sample in front of G1 changes the average intensity (absorption signal), the phase shift (phase signal) and the amplitude (scattering signal) of those fringes. Since the fringe period is too small (thus ranging between 2 up to $10 \mu \mathrm{m}$, depending on the grating period) with respect to the typical detector pixel size (in a lab setting, it is in the order of 50-70 $\mu \mathrm{m}$ ), the analyzer grating G2 is used to detect those changes. The phase-stepping [11] and Moiré-fringe [12, 23] methods are the most known GI acquisition protocols. In the phase-stepping approach, G2 is moved in uniform steps in the transversal beam direction and an image is acquired at each position. A reference (without sample) and an object phasestepping scan must be acquired to retrieve the relative differences in the signals. From the phase-stepping curves (PSC), available in each pixel, the three signals (ABS, DPC and DF) are calculated via a Fourier coefficient analysis [24].

The Moiré fringe method consists in the detection of the reference fringe pattern by observing the Moiré fringes, which are generated on the detector plane when the G2 and G1 gratings are tilted by a small angle. The superposition of periodic structures generated by the two gratings rotated towards each other leads to a periodic pattern, called Moiré pattern [25], with a period larger than the pixel size. An object in front of G1 will cause deformations of the Moiré pattern. The information about ABS, DPC and DF can then be extracted from the Fourier Transform of the measured Moiré pattern image [26, 27]. The Moiré imaging approach slightly worsens the spatial resolution with respect to the phase stepping, but it is 


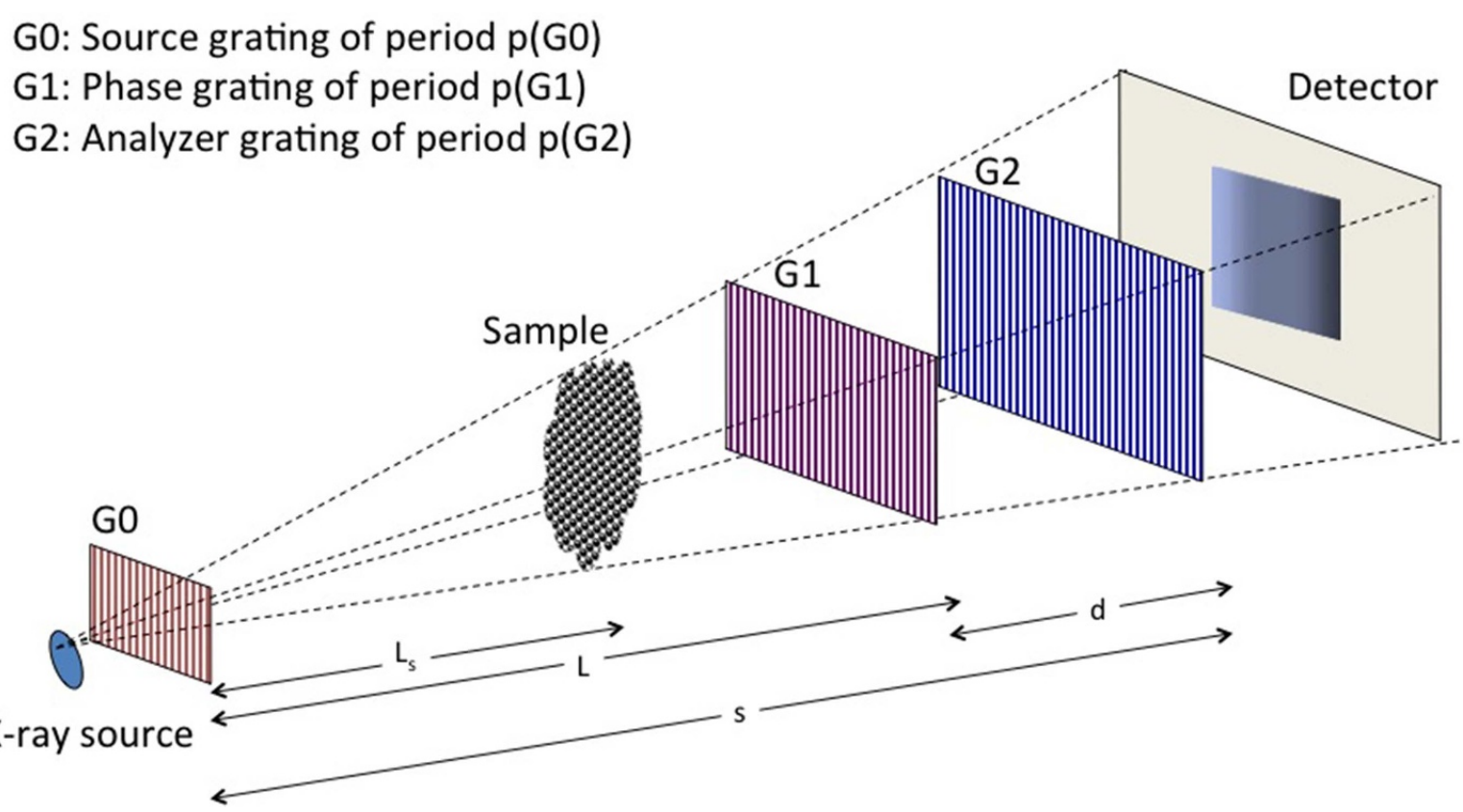

Figure 1. GI scheme. The main components are: an x-ray source and a detector and a set of three gratings (G0, G1 and G2) located in between with the sample in front of G1. $\mathrm{L}_{\mathrm{s}}$ is the $\mathrm{G}_{0}$-sample distance; $\mathrm{L}$ is the $\mathrm{G}_{0}-\mathrm{G}_{1}$ distance; $\mathrm{d}$ is the inter-grating distance and $\mathrm{s}$ is the total system arrangement.

still compliant with the requirements for medical applications such as lung imaging [28].

Two figures of merit define the performance of a gratings system: the visibility and the sensitivity. The visibility (V) is the normalized magnitude of the first Fourier component of the PSC. For a harmonic oscillation pattern, the visibility is defined as the oscillation amplitude normalized to the mean intensity. By quantifying the loss of visibility it is possible to obtain the contrast denoted as dark-field contrast. In Pfeiffer et al [29] and Yashiro et al [30], the visibility contrast is expressed through the mechanism of small-angle x-ray scattering from microstructures with a scale much smaller than the spatial resolution of the imaging system and it is formulated by an autocorrelation function describing the spatial fluctuations of a wave-front due to the microstructures. The GI sensitivity is inversely related to the smallest detectable refraction angle $\alpha_{\min }$ [13], which is proportional to the smallest detectable interference fringe shift, $\varphi_{\min }$, according to [31]:

$$
\alpha_{\min }=\frac{p(G 2)}{2 \pi d} \frac{L}{L_{\mathrm{s}}} \varphi_{\min }
$$

where $\mathrm{p}(\mathrm{G} 2)$ refers to the pitch of the analyzer grating $\mathrm{G} 2$ and $d$ is the inter-grating distance between $\mathrm{G} 1$ and G2, defined as:

$$
d=s \frac{\eta p(G 2)-p(G 1)}{\eta p(G 2)}
$$

In equation (2), $s$ is the total setup length (i.e. source to detector distance, if the detector is located as close as possible to G2), and $\eta$ indicates whether the beam splitter grating is a $\pi / 2$ shifting $(\eta=1)$ or a $\pi$-shifting $(\eta=2)$ phase grating.
The phase-contrast images quality in GI is quantified in terms of the SNR. Both signal and noise depend on the geometric parameters while the signal depends on the object as well. This implies that SNR can be defined as:

$$
\operatorname{SNR}_{\varphi}=\alpha \frac{2 \pi d}{p(G 2)} \frac{V \sqrt{N}}{\sqrt{2 X_{\mathrm{det}}}}
$$

where $\alpha$ is the refraction angle. The $\alpha$ standard deviation $\sigma_{\alpha}$ defined as:

$$
\sigma_{\alpha}=\frac{p(G 2)}{2 \pi d} \frac{\sqrt{2 X_{\mathrm{det}}}}{V \sqrt{N}}
$$

corresponds to the smallest detectable refraction angle. On the other hand, $\sigma_{\alpha}$ depends linearly on the number of counts $\mathrm{N}$ and on the visibility V $[32,33]$ and inversely on $X_{d e t}$, the number of analog-to-digital units per incoming photon. The proportionality of $\sigma_{\alpha}$ and $\frac{p_{(G 2)}}{d}$ suggests that an optimization can be achieved by using a small analyzer grating pitch and long experimental arrangements, with higher Talbot order designs.

To construct a highly performing GI system, all these parameters require an optimization.

The simulation pipeline is split into two parts. First, the $\mathrm{x}$-ray spectrum simulations, performed with the SpekCalc software [34], were validated by measurements of air kerma with an ion chamber, as discussed in section D. In the second part, based on a wave propagation code written in MATLAB $[35,36]$, we simulated all the relevant system parameters, taking into account the laboratory setup and the geometric constraints, and selected the configuration corresponding to the best-expected performance. In the last part of this study, the energy spectra at the different propagation stages are simulated with the GATE [37] open source package. 


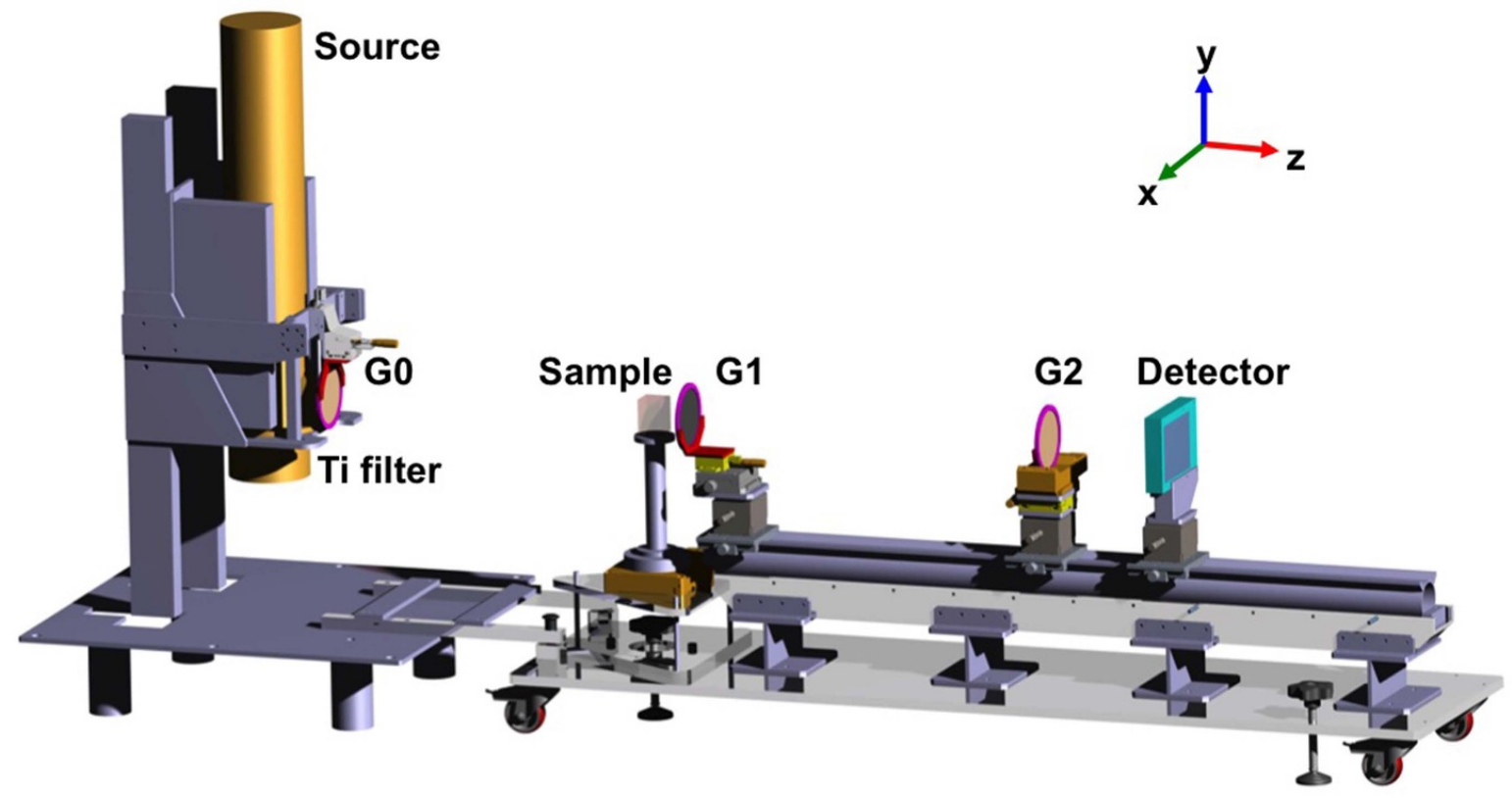

Figure 2. Schematic view of the 4D GRAPH-X system. All the components are equipped with their motors as specified in the text.

\subsection{System configuration}

The 4D GRAPH-X device for X-ray phase contrast imaging based on TL GI is installed in a radiation-protected area of the Physics Department at the University of Torino, Italy.

The system, shown in figure 2 , consists of a source, a detector and a set of three gratings: G0, G1 and G2. A sample is located between G0 and G1. The x-ray source is an ordinary GE Eresco 160 MF4-R x-ray tube with a millimetric focal spot size and tungsten anode. The maximum voltage, current and power are $160 \mathrm{kV}, 10 \mathrm{~mA}$ and $1600 \mathrm{~W}$, respectively.

The $\mathrm{x}$-ray tube is air-cooled for ease of transportation. A $0.5 \mathrm{~mm}$ thick Titanium filter is inserted in front of the source in order to model the energy spectrum with a peak around the design energy (i.e. $45 \mathrm{keV}$ at $85 \mathrm{kVp}$ ). The detector is a flat panel from Shad-o-Box 6 K HS by Teledyne Dalsa, with a CMOS active part with a $114 \times 146 \mathrm{~mm}^{2}$ sensitive area and a $49.5 \mu \mathrm{m}$ pixel pitch. It is positioned directly after G2 on a linear stage platform able to perform translations along the beam direction together with G1 and G2.

The design optimization study is extensively discussed in the results section. The grating specifications (i.e. inter-grating distances and grating parameters) have been determined, by means of wave field simulations, to maximize the device performance in terms of visibility and sensitivity. The total setup length refers to the G0-G2 distance, whereas gratings were designed for a specific energy, which corresponds to the energy of the incoming spectrum that guarantees the best achievable GI performance.

In this configuration, the G0 grating is equipped with a manually controlled linear XYZ stage to adjust its position along the three main axes. The G1 grating is mounted on a motorized linear stage for precise alignment with a $100 \mathrm{~nm}$ minimum incremental motion, transversal to the beam direction, in order to perform a phase stepping acquisition protocol. However, since phase stepping is a long acquisition method, thus not suitable for medical applications, Moiré imaging is implemented as well. For this reason, the G2 grating is equipped with a motorized goniometer with a minimum incremental motion of 0.2 mdeg. G1, G2 and the detector are located on linear stages for the fine alignment of the vertical position. A rail system between the phase and the analyzer grating allows a travel range up to $1.5 \mathrm{~m}$, in order to increase the system sensitivity at higher Talbot orders. The maximum achievable grating size based on $100 \mu \mathrm{m}$ wafer micro-fabrication technology is about $7 \times 7 \mathrm{~cm}^{2}$. This size constrains the system field of view (FOV), while the spatial resolution is limited by the Moiré imaging approach, the grating pitch and the pixel size of the detector.

\subsection{Test specimen}

As a specimen, a $3 \mathrm{~cm}$ thick and $5 \mathrm{~cm}$ long anthropomorphic dynamic thorax phantom similar to the one described in [38] is currently under construction in collaboration with the University of Florence (figure 3 ). The phantom simulates a male torso containing moving structures capable of reproducing realistic lung lesions movements. While the external phantom surface is $3 \mathrm{D}$ printed using as reference a real patient $\mathrm{CT}$ acquisition (and then rescaled to a small phantom size to be suitable with the 4D GRAPH-X system), internal parts are made with materials mimicking lungs, muscles and ribs density and attenuation. Water equivalent inserts (WEIs) simulating spherical tumors can be positioned into the lungs in different locations. An Arduino programmable board drives a step-motor to move the spheres along linear paths. 
(a)

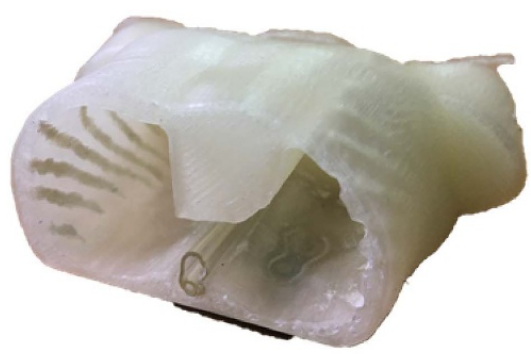

(b)

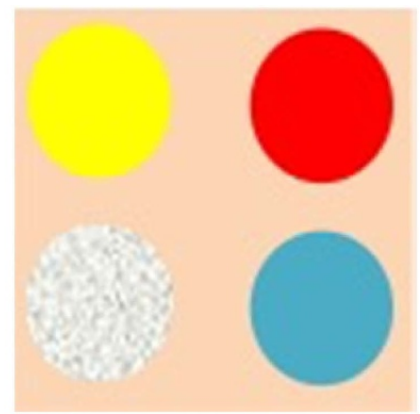

Figure 3. External (a) and simplified internal (b) view of the phantom. The existing ADAM phantom will be rescaled (junior version named ADAM J.) to fit within the FOV of the proposed 4D GRAPH-X prototype. The internal part will contain lungs (yellow), muscles (red), ribs (grey) and WEI (blue).

\subsection{Source simulation and validation}

The SpekCalc software is based on Monte Carlo simulations to determine the energy and depth distribution of electrons in a tungsten $\mathrm{x}$-ray target and includes a semi-relativistic expression for the Bethe-Heitler Bremsstrahlung cross section differential in energy $[34,39,40]$. It requires some input parameters, such as peak energy, filter thickness and a normalization factor for the overall fluence $\mathrm{N}_{\mathrm{f}}$ necessary to match the output prediction to that of a particular tube. The $\mathrm{N}_{\mathrm{f}}$ correct value is obtained by normalizing the simulated spectrum to the measured one in terms of air kerma. Measurements of air kerma are performed using a $6 \mathrm{~cm}^{3}$ ion chamber combined with the Radcal Model 9015 [41].

\section{Results}

\section{1. $x$-ray source and gratings specifications}

Table 1 lists the most important beam qualifiers:

- the homogeneity coefficient for both $\mathrm{Al}$ and $\mathrm{Cu}$, defined as HVL1/ HVL2 [34], where HVL1 (2) is the first (second) half value layer, i.e. the thickness of material required to reduce the intensity of an X-ray beam to half its initial value; it indicates to what extent the filtration has produced an homogeneous beam close to mono-energetic conditions $(\sim 1)$;

- the spectrum mean energy, defined as the beam energy averaged over the fluence spectrum; the estimated bremsstrahlung and characteristic contributions to the tube output ( $\mu \mathrm{Gy} / \mathrm{mAs} @ 1 \mathrm{~m})$.

The x-ray attenuation caused by the gratings is calculated for the spectrum specified in table 1 and considering the attenuation coefficients of the grating fabrication materials (Au for G0 and G2; Si for G1). Results referring to gratings with a duty cycle of 0.5 are reported in figure 4 . The characteristic peaks of the tungsten (W) K series are visible between 57 and $70 \mathrm{keV}$.

For a $45 \mathrm{keV}$ beam, the transmission of the substrate is approximately $47 \%$, which is equivalent to a $350 \mu \mathrm{m}$ thick
Table 1. SpekCalc results for a tube at $85 \mathrm{kV}$ energy and with a $0.5 \mathrm{~mm}$ Titanium filter at $1 \mathrm{~m}$ distance.

\begin{tabular}{ll}
\hline Mean energy $[\mathrm{keV}]$ & 47 \\
HVL1/HVL2 $(\mathrm{Al})$ & 0.7 \\
HVL1/HVL2 $(\mathrm{Cu})$ & 0.6 \\
Bremsstrahlung output $[\mu \mathrm{Gy} / \mathrm{mAs}]$ & 133.9 \\
Characteristic output $[\mu \mathrm{Gy} / \mathrm{mAs}]$ & 2.845 \\
\hline
\end{tabular}

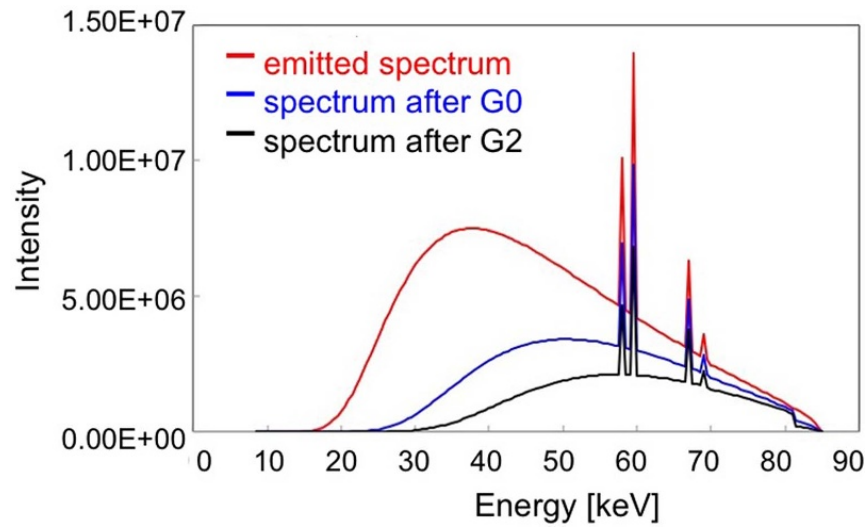

Figure 4. Attenuated spectrum after gratings. In particular, the gold gratings G0 and G2 are considered. Since G1 is in silicon, a low absorbing material, the spectrum after G1 nearly overlaps to the spectrum after G0.

Si substrate. Although only $21 \%$ of photons are transmitted after passing through all gratings, the visibility is still high enough to ensure good image quality (for the selected parameters, the visibility is about $22 \%$, as discussed in the next paragraph). This would correspond to an increase in scan time by a factor of 3.3 compared to standard absorption imaging with the same source equipped by a titanium filter and without any gratings. Another important factor is the dose increment for an absorption image with the GI to achieve the same SNR as with a standard absorption imaging setup without a GI. The calculated total transmission after the gratings indicates that a dose increase by a factor $\frac{1}{\sqrt{0.21}}=2.2$ would be required for the GI system to obtain the same SNR of a conventional 
absorption-based x-ray CT. This factor matches the theoretical prediction [42], whereas the benefit for GI compared with conventional CT is obtained in the case of a maximum dose efficiency bigger than 1 . The G0 and G2 grating heights are chosen to maintain high absorbance beam percentage (close to $100 \%$ of the beam) while the $\mathrm{G} 1$ grating height $\left(\mathrm{h}_{1}\right)$ is calculated in order to obtain a $\pi$ phase shift:

$$
h_{1}=\frac{\pi}{\lambda \rho r_{0}}=1.28[\mu \mathrm{m} / \mathrm{keV}] \times E[\mathrm{keV}]
$$

where $r_{0}=2.82 \times 10^{-5} \dot{A}$ is the Thomson scattering length and $\rho$ is the electron density: for silicon, $\rho_{S i}=0.70 \dot{A}^{-3}$. A schematic representation of the grating structure is reported in [43]. With $45 \mathrm{keV} x$-ray energy, the height of the G1 grating must be $58 \mu \mathrm{m}$ to produce a $\pi$ phase shift.

It is also important to point out that in a compact arrangement, a large beam divergence enables a large FOV, even if the geometrical magnification is high. However, in GI a high beam divergence leads to high aspect ratios (ARs) of the gratings [44]. The AR, defined as the structure height $(h)$ divided by half a pitch $(p)$ or line-width:

$$
\mathrm{AR}=2 \frac{h}{p}
$$

can be arbitrarily large. In particular for the absorption grating, where the structure's heights must be as large as possible, AR is extremely high (e.g. up to 100). This alters the grating transmission function at beam-incident angles close to or higher than 1/AR, reducing the visibility of the phase-stepping curve and preventing signal retrieval, leading to a reduction of the FOV (the so-called shadow effect). In particular, the shadow effect should be considered to obtain an estimation of the expected effective area defining the FOV when the x-ray energy is high. In [45], this effect is discussed and the FOV is evaluated by:

$$
\begin{gathered}
\tan \theta=\frac{\frac{\mathrm{FOV}}{2}}{s}=\frac{w}{h} \\
\mathrm{FOV}=\frac{2 w s}{h}
\end{gathered}
$$

where $w$ is the trench width, $h$ is the Au height of the grating, $s$ is the total distance between source and detector (figure 5). In the symmetric geometry, $w$ and $h$ are designed to be the same values for both $\mathrm{G} 0$ and $\mathrm{G} 2$, leading to equivalent shadow effects.

To reduce such an effect, a solution could be to bend the gratings by a properly defined angle.

In this work, to avoid unnecessary bending while providing a better contrast, the design energy is set to $45 \mathrm{keV}$. Indeed, the achievable AR of the Au gratings is limited to 34:1, due to the gratings height required at such energy. This value reduces the problem of the limited FOV, so that gratings' curving is less critical. Smaller pitch or higher structures with higher ARs can be achieved in terms of fabrication [46].

In addition, the average energy of the $\mathrm{x}$-ray spectrum, with voltage set to $85 \mathrm{kV}$ and a titanium filter, is close to the design

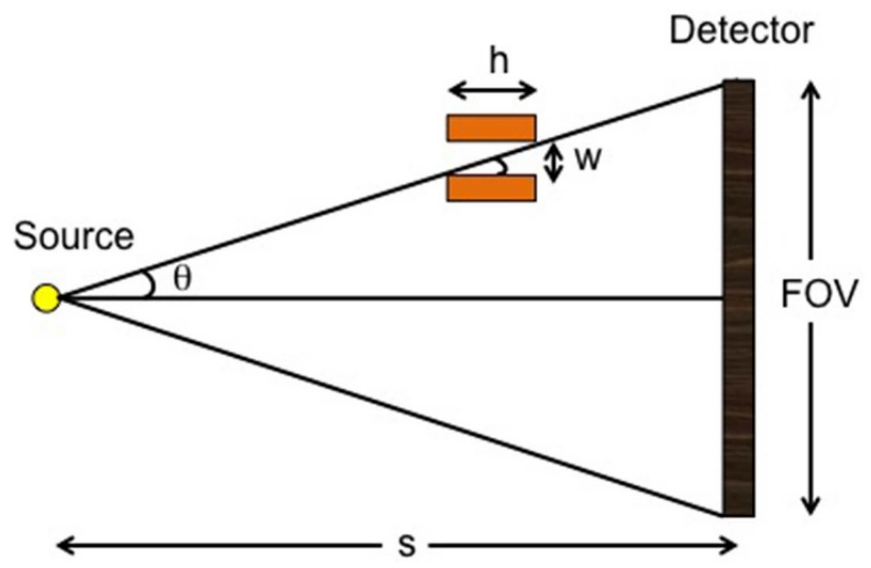

Figure 5. Description of the shadow effect. The effective area depends on the FOV and grating diameter. Reprinted by permission from Springer Nature Customer Service Centre GmbH: Springer Nature. The Korean Physical Society, [45].

Table 2. Case study at $45 \mathrm{keV}$ design energy.

\begin{tabular}{lr}
\hline $\mathrm{h}_{0,2}[\mu \mathrm{m}]$ & 90 \\
$\mathrm{~h}_{1}[\mu \mathrm{m}]$ & 58 \\
Design energy $[\mathrm{keV}]$ & 45 \\
\hline
\end{tabular}

energy (figure 4, red line). This spectrum modulation is carefully chosen to avoid a dramatic decrease of the number of photons.

\subsection{GI setup optimization}

The optimization of the GI setup is performed with a MATLAB program based on numerical simulations. The system visibility and sensitivity are calculated in order to assess the expected performance. As input to these simulations, the x-ray spectrum plotted in figure 4 (red line) is considered while the selected grating heights and design energy are summarized in table 2. To better assess the system performance, simulations are done at different values of the Talbot orders (T.O.): first (1), third (3) and fifth (5).

The spectral visibility responses at $1.5 \mathrm{~m}$ source-to-detector distance at the three T.O. are shown in figure 6. The mean visibility is of the same order of magnitude in the first three T.O. (specifically, 1: 25\%, 3: 22\%; 5: 24\%). The main sources of errors depend not only on the photon statistics but also on the number of phase steps, the actual phase shift and the interferometer type. The error is higher for a TL type interferometer compared to a $\mathrm{T}$ type interferometer with similar coherence properties. In a TL type, it is recommended to acquire at least seven steps to keep the error below $1 \%$. Additionally, an odd number of steps should be acquired in order to reduce the error on the measured visibility.

In this work, the sensitivity is approximated to the inverse of the smallest refractive angle in equation (4). In order to get a trend for the first three Talbot orders, the ratio $\frac{p(G 2)}{d}$ is plotted in figure 7 . Such a ratio provides an estimation for the 4D GRAPH-X system sensitivity (referred as $\Sigma^{-1}$ in the plot). 

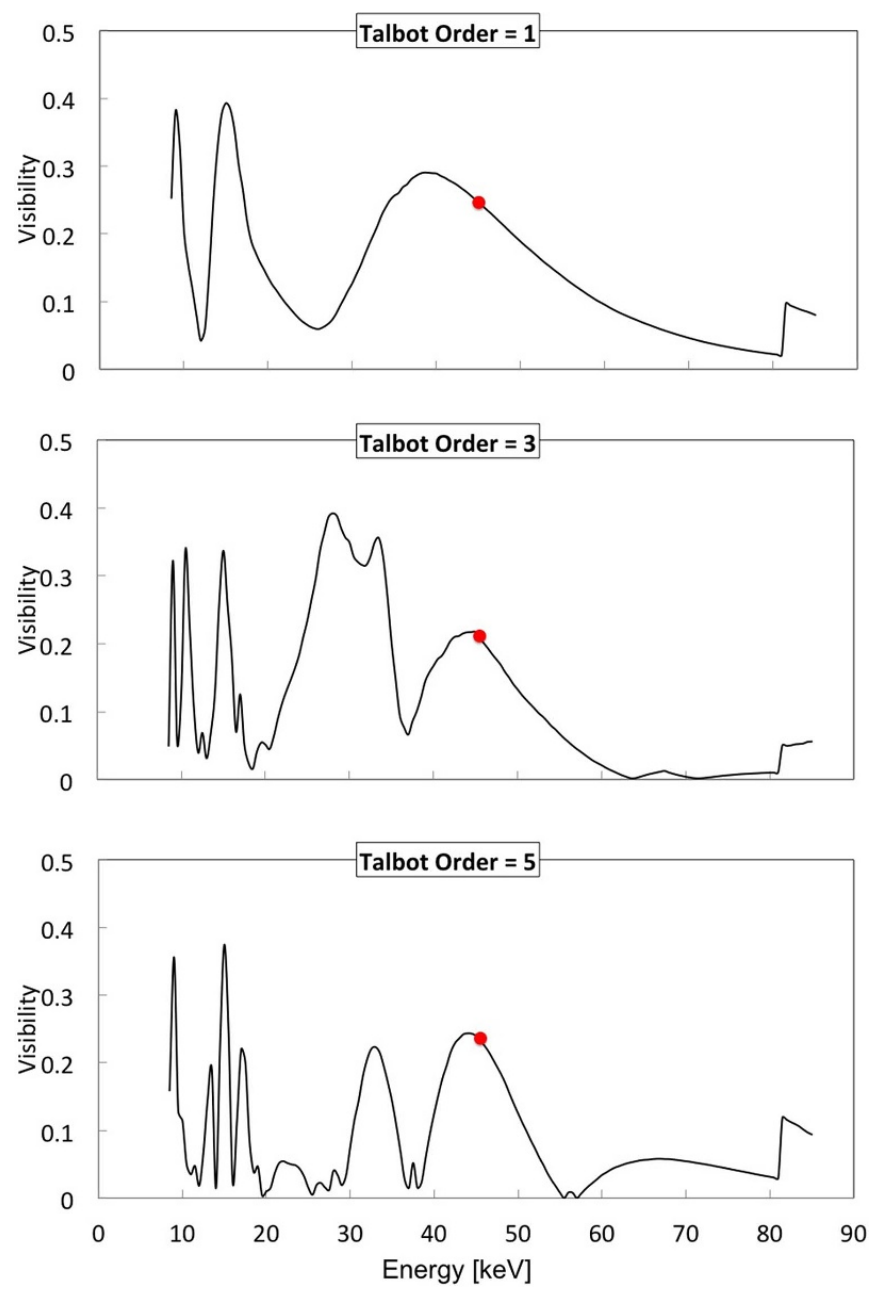

Figure 6. Spectral visibility as a function of the energy for T.O. $=1,3,5$. Gratings pitch: $5.25 \mu \mathrm{m}$ at $45 \mathrm{keV}$ energy.

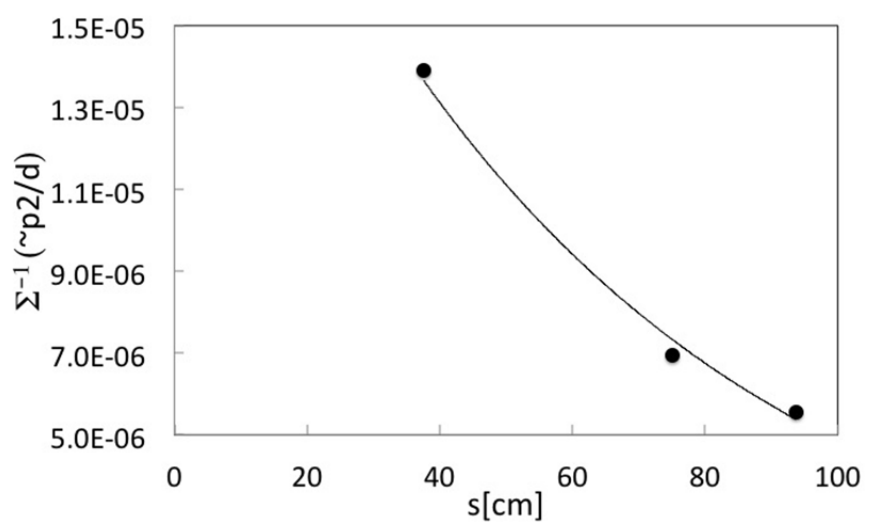

Figure 7. A trend for the system sensitivity $\left(\Sigma^{-1}\right)$ as a function of G1-G2 distance (s) at the three T.O. investigated in this work.

While the sensitivity slightly improves at higher Talbot orders and so at larger inter-grating distances (figure 7), a symmetric configuration is preferable to characterize the system in order to facilitate GI manufacture and installation procedure.

Indeed, a symmetric configuration with equal grating distances and pitches for G0 and G2 implies a similar
Table 3. Grating configurations with a fixed analyzer grating pitch of $5.25 \mu \mathrm{m}$.

\begin{tabular}{|c|c|c|c|}
\hline T.O./[ $[\mu \mathrm{m}]$ & $\mathrm{p}(\mathrm{G} 0)$ & $\mathrm{p}(\mathrm{G} 1)$ & $\mathrm{p}(\mathrm{G} 2)$ \\
\hline 1 & 15.75 & 7.87 & 5.25 \\
\hline 3 & 5.25 & 5.25 & 5.25 \\
\hline 5 & 3.15 & 3.93 & 5.25 \\
\hline
\end{tabular}

fabrication, as the structure height is the same, and thus the implementation of the system is straightforward. Therefore, the optimum selection is with equal values of the two gratings pitches, yielding a symmetric interferometer arrangement obtained at a total length of 1.5 meters and at the third Talbot order (as seen in table 3 , which reports the gratings parameters resulting from the simulations).

The pitch is selected by taking into account both the simulation results and the available fabrication technology, in order to guarantee the optimal grating performance at the desired energy (thus pushing to a height of $90 \mu \mathrm{m}$ ). In addition to that, the total system length is adapted to the laboratory limitations in terms of available space where the setup is installed.

The total setup length is set to $150 \mathrm{~cm}$ and the G0 and G2 gold gratings trench width and height are set to $2.625 \mu \mathrm{m}$ and $90 \mu \mathrm{m}$, respectively. The resulting FOV, calculated as in equation (8), is almost $9 \mathrm{~cm}$ wide, comparable to the sample size.

\subsection{Lung imaging-preliminary study}

For the evaluation of the x-ray fluence, the spectrum parameters listed in table 1 are given as input to the wavefront based simulation code.

The optimized grating parameters (tables 2 and 3) are used in the Monte Carlo simulation performed with GATE, so as to evaluate the system attenuation and transmission. A body phantom is simulated as a box of $7 \times 7 \times 3 \mathrm{~cm}^{3}$ volume containing four spheres respectively of lung $\left(\rho=0.26 \mathrm{~g} \mathrm{~cm}^{-3}\right)$, muscle $\left(\rho=1.05 \mathrm{~g} \mathrm{~cm}^{-3}\right)$, rib bones $\left(\rho=1.92 \mathrm{~g} \mathrm{~cm}^{-3}\right)$ and water equivalent $\left(\rho=1.00 \mathrm{~g} \mathrm{~cm}^{-3}\right)$ inserts.

The entire phantom fits the beam with an emission angle of $6^{\circ}$. The gold source grating (G0) is inserted $6 \mathrm{~cm}$ downstream of the source center, with $90 \mu \mathrm{m}$ height, $7 \times 7 \mathrm{~cm}^{2}$ mask and $5.25 \mu \mathrm{m}$ pitch. The silicon phase grating (G1) is inserted $75 \mathrm{~cm}$ downstream of the source center, with $58 \mu \mathrm{m}$ height, $7 \times 7 \mathrm{~cm}^{2}$ mask and $5.25 \mu \mathrm{m}$ pitch. Finally, the gold analyzer grating (G2) is inserted $150 \mathrm{~cm}$ downstream of the source center, with $90 \mu \mathrm{m}$ height, $7 \times 7 \mathrm{~cm}^{2}$ mask and $5.25 \mu \mathrm{m}$ pitch.

The simulation process is sketched in figure 8. In figure 9, the energy spectra after the source, the source grating, the phantom, the phase grating and the analyzer grating are shown. The corresponding statistics are summarized in table 4 . Results by Monte Carlo simulations confirm the analytical calculations. After passing through the G0 source grating, the transmission percentage is equivalent to about $56 \%$ (compared to $47 \%$ obtained by numerical simulation). The transmission after the phantom is about $33 \%$. Finally, the transmission after 


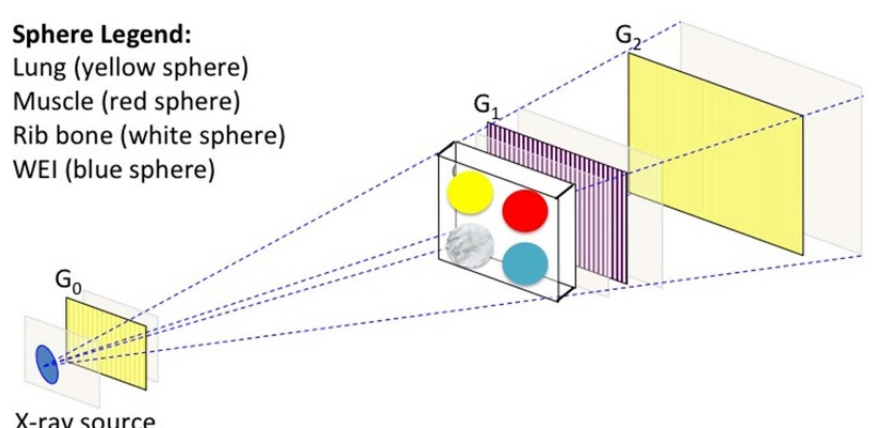

X-ray source

Figure 8. GATE Monte Carlo simulation visualization. five simulated detectors (transparent gray) are located beside the source, G0, the sample, G1 and G2 in order to calculate the energy spectrum in 5 different positions.

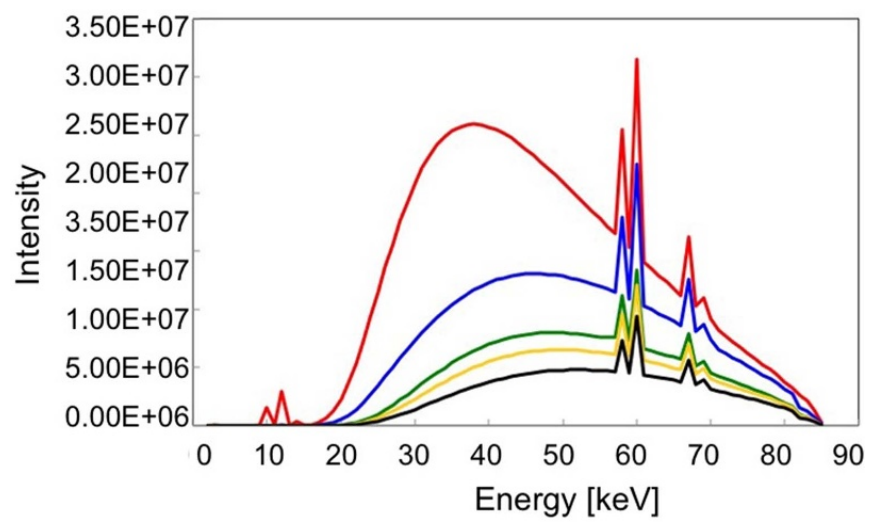

Figure 9. Energy spectra detected after (red) x-ray source, (blue) source grating, (green) phantom, (yellow) phase grating and (black) analyzer grating.

Table 4. Number of incoming photons (i.e. photons/unit time/unit area), mean energy ( $\left.E_{\text {mean }}\right)$ and standard deviations.

\begin{tabular}{llcc}
\hline & Entries & $\mathrm{E}_{\text {mean }}(\mathrm{keV})$ & Std.Dev. $(\mathrm{keV})$ \\
\hline X-ray & $1.00 \mathrm{E}+09$ & 46 & 15 \\
G0 & $5.57 \mathrm{E}+08$ & 51 & 14 \\
Phantom & $3.27 \mathrm{E}+08$ & 52 & 13 \\
G1 & $2.69 \mathrm{E}+08$ & 53 & 13 \\
G2 & $1.98 \mathrm{E}+08$ & 54 & 13 \\
\hline
\end{tabular}

the G2 analyzer grating is about $20 \%$. Small discrepancies in transmission percentages obtained with the numerical and MC methods could be related to slight differences in the x-ray emission angle. The MC simulation is more reliable for two reasons: a more realistic sample is introduced and, also, the emission angle is better selected in order to cover the full visible area.

As the spectrum of photon energies in an x-ray beam is wide, low-energy photons are much more likely than high-energy photons to be absorbed. As a consequence, the higher energy photons are more easily transmitted. Therefore as the $\mathrm{x}$-rays pass through each component, the intensity of the beam decreases while its average energy increases (see table 4).

\section{Discussion and conclusion}

In this work, the implementation of an x-ray grating-based phase-contrast imaging scanner based on a single-shot acquisition method, is presented. The design optimization procedure is performed by means of wavefront simulations with numerical and MC based codes. Results show that the optimal GI design is obtained for a symmetric configuration with $5.25 \mu \mathrm{m}$ gratings pitch and $45 \mathrm{keV}$ energy, at the third T.O. The calculated FOV of $9 \mathrm{~cm}$ width is suitable for a small phantom that simulates the chest composition (i.e. containing spheres with densities similar to the lungs, rib bones, muscle and a WEI) and respiratory cycle. Monte Carlo simulations provide an estimate of the x-rays attenuation after the phantom. The beam attenuation is investigated by inserting a virtual screen after each optical element of the system and after the sample; however, no simulations in phase contrast modality are performed in GATE. The source is simulated with a $6^{\circ}$ emission angle.

The $22 \%$ visibility expected with the simulated polychromatic source will be validated during laboratory tests, as noise in phase contrast imaging is highly dependent on the visibility. The system parameters are tuned in order to investigate the potential of the setup for dynamic imaging in view of future applications in lung imaging. The designed system aims at demonstrating the diagnostic power of phase contrast $\mathrm{x}$-ray imaging in a laboratory setting. Furthermore, the system optimization is studied for a flexible design in order to be applied in a future study for a dual scope: in an initial configuration, with the $9 \mathrm{~cm}$ width FOV for the high-resolution virtual biopsy of suspicious regions and, later on, by increasing the FOV by means of stitched gratings in order to investigate the entire lung area. On the other side, the choice of certain parameters, such as energy or grating pitch, is strategic in view of future applications in food science or cultural heritage. The construction is now under way.

\section{Data availability statement}

All data that support the findings of this study are included within the article (and any supplementary files).

\section{Acknowledgments}

This work is supported by the INFN Group 5 Young Investigators Grant 4D GRAPH-X. We acknowledge the NEXTO project (progetti di Ateneo 2017), funded by Compagnia di San Paolo, and the INFN mechanical services, in particular Giuseppe Scalise who provided the mechanical drawing of the system. Marco Stampanoni (Paul Scherrer Institute, Switzerland), Vincenzo Monaco (University of Torino, Italy) and Luigi Rigon (University of Trieste, Italy) are acknowledged for insightful discussions. 


\section{ORCID iDs}

Alessandra Patera (D) https://orcid.org/0000-0001-5760-3118 Stefania Pallotta (D) https://orcid.org/0000-0003-4123-7971

Luciano Ramello (D) https://orcid.org/0000-0003-2325-8680

Alessandro Re (D) https://orcid.org/0000-0001-9952-5488

\section{References}

[1] Momose A 2005 Recent advances in x-ray phase imaging Jpn. J. Appl. Phys. 44 6355-67

[2] Als-Nielsen J and McMorrow D 2011 Elements of Modern $x$-ray Physics (New York: Wiley)

[3] Momose A 1995 Demonstration of phase contrast x-ray computed tomography using an x-ray interferometer $\mathrm{Nucl}$. Instrum. Methods Phys. Res. A 352 622-8

[4] Kitchen M et al 2017 CT dose reduction factors in the thousands using x-ray phase contrast Sci. Rep. 715953

[5] Bonse U and Hart M 1965 An x-ray interferometer Appl. Phys. Lett. 6 155-6

[6] Snigirev A, Snigireva I, Kohn V, Kuznetsov S and Schelokov I 1995 On the possibilities of x-ray phase contrast microimaging by coherent high-energy synchrotron radiation Rev. Sci. Instrum. 66 5486-92

[7] Momose A, Takeda T, Itai Y and Hirano K 1996 Phase contrast X-ray computed tomography for observing biological soft tissues Nat. Med. 2 473-5

[8] Chapman D, Thomlinson W, Johnston R, Washburn D, Pisano E, Gmür N, Zhong Z, Menk R, Arfelli F and Sayers D 1997 Diffraction enhanced x-ray imaging Phys. Med. Biol. 422015

[9] Olivo A et al 2001 An innovative digital imaging set-up allowing a low-dose approach to phase contrast applications in the medical field Med. Phys. 28 1610-9

[10] David C, Nöhammer B, Solak H H and Ziegler E 2002 Differential x-ray phase contrast imaging using a shearing interferometer Appl. Phys. Lett. 81 3287-9

[11] Weitkamp T et al $2005 \mathrm{x}$-ray phase imaging with a grating interferometer Opt. Express 13 6296-304

[12] Momose A, Kawamoto S, Koyama I, Hamaishi Y, Takai K and Suzuki Y 2003 Demonstration of X-ray talbot interferometry Jpn. J. Appl. Phys. 42 L866

[13] Pfeiffer F, Weitkamp T, Bunk O and David C 2006 Phase retrieval and differential phase contrast imaging with low-brilliance x-ray sources Nat. Phys. 2 258-61

[14] Bech M et al 2013 In-vivo dark-field and phase contrast x-ray imaging Sci. Rep. 33209

[15] Rigon L, Besch H J, Arfelli F, Menk R-H, Heitner G and Plothow-Besch H 2003 A new DEI algorithm capable of investigating sub-pixel structures J. Phys. D: Appl. Phys. 36 A $107-\mathrm{A} 112$

[16] Yaroshenko A, Hellbach K, Bech M, Grandl S, Reiser M F, Pfeiffer F and Meinel F G 2014 Grating-based x-ray dark-field imaging: a new paradigm in radiography $\mathrm{Curr}$. Radiol. Rep. 257

[17] Stampanoni M, Wang Z, Thüring T, David C, Roessl E, Trippel M, Kubik-Huch R A, Singer G, Hohl M K and Hauser N 2011 The first analysis and clinical evaluation of native breast tissue using differential phase contrast mammography Invest. Radiol. 46 801-6

[18] Michel T et al 2013 On a dark-field signal generated by micrometer-sized calcifications in phase contrast mammography Phys. Med. Biol. 582713

[19] Willer K et al 2018 x-ray dark-field imaging of the human lung: a feasibility study on a deceased body PLoS One $13 \mathrm{e} 0204565$
[20] Scherer K et al 2017 x-ray dark-field radiography-in vivo diagnosis of lung cancer in mice Sci. Rep. 7402

[21] Gromann L B et al 2017a In vivo X-ray dark-field chest radiography of a pig Sci. Rep. 74807

[22] Gromann L B et al 2017b First experiences with in vivo x-ray dark-field imaging of lung cancer in mice SPIE Medical Imaging (International Society for Optics and Photonics) p 101325L

[23] Momose A, Yashiro W, Maikusa H and Takeda Y 2009 Phase-sensitive imaging and phase tomography using x-ray interferometers Opt. Express 17 12540-5

[24] Thüring T et al 2014 Performance and optimization of x-ray grating interferometry Phil. Trans. A 37220130027

[25] Amidror I 2009 The Theory of the Moiré Phenomenon-Volume I: Periodic Layers (Berlin: Springer)

[26] Bennett E E, Kopace R, Stein A F and Wen H 2010 A grating-based single-shot $\mathrm{x}$-ray phase contrast and diffraction method for in vivo imaging Med. Phys. 37 6047-54

[27] Bevins N, Zambelli J, Li K, Qi Z and Chen G H 2012 Multicontrast X-ray computedtomography imaging using Talbot-Lau interferometry without phase stepping Med. Phys. 39 424-8

[28] Sefert M et al 2018 Improved reconstruction technique for moiré imaging using an X-ray phase-contrast Talbot-Lau interferometer J. Imaging 462

[29] Bech P M, Bunk O, Kraft P, Eikenberry E F, Brönnimann C H, Grünzweig C and David C 2008 Hard-x-ray dark-field imaging using a grating interferometer Nat. Mater. 7 134-7

[30] Yashiro W, Terui Y, Kawabata K and Momose A 2010 On the origin of visibility contrast in X-ray Talbot interferometry Opt. Express 18 16890-901

[31] Thüring T, Modregger P, Hämmerle S, Weiss S, Nüesch J and Stam- Panoni M 2012 Sensitivity in X-ray grating interferometry on compact sys- tems AIP Conf. Proc. $1466293-8$

[32] Engel K J, Geller D, Köhler T, Martens G, Schusser S, Vogtmeier G and Rössl E 2010 Contrast-to-noise in x-ray differential phase contrast imaging Nucl. Instrum. Methods Phys. Res. A 648 202-7

[33] Revol V, Kottler C, Kaufmann R, Straumann U and Urban C 2010 Noise analysis of grating-based x-ray differential phase contrast imaging Rev. Sci. Instrum. 81073709

[34] Poludniowski G et al 2009 SpekCalc: a program to calculate photon spectra from tungsten anode x-ray tubes Phys. Med. Biol. 54 N433-N438

[35] Thüring T 2013 Compact x-ray grating interferometry for phase and dark-field computed tomography in the diagnostic energy range $E T H P h D$ thesis

[36] Arboleda C 2017 Development of a phase contrast clinical mammography prototype for the in-vivo investigation of breast cancer $E T H P h D$ thesis

[37] Sébastien J 2011 GATE V6: a major enhancement of the GATE simulation platform enabling modelling of CT and radiotherapy Phys. Med. Biol. 56 881-901

[38] Pallotta S et al 2018 ADAM: a breathing phantom for lung SBRT quality assurance Phys. Med. 49 147-55

[39] Poludniowski G G and Evans P M 2007 Calculation of X-ray spectra emerging from an $\mathrm{x}$-ray tube. Part I. Electron penetration characteristics in x-ray targets Med. Phys. 34 2164-74

[40] Poludniowski G G 2007 Calculation of x-ray spectra emerging from an X-ray tube. Part II. X-ray production and filtration in x-ray targets Med. Phys. 34 2175-86

[41] Bogard J S 1998 RADCAL operations manual radiation calibration laboratory protocol, United States Department of Energy, Office of Energy Research 
[42] Raupach R and Flohr T 2012 Performance evaluation of x-ray differential phase contrast computed tomography (PCT) with respect to medical imaging Med. Phys. 39 4761-74

[43] Shi Z, Jefimovs K, Romano L and Stampanoni M 2020 Towards the fabrication of high-aspect-ratio silicon gratings by deep reactive ion etching Micromachines $\mathbf{1 1} 864$
[44] Thüring T et al $2014 \mathrm{x}$-ray phase-contrast imaging at $100 \mathrm{keV}$ on a conventional source Sci. Rep. 45198

[45] Lee $\mathrm{S}$ et al 2018 System design and evaluation of a compact and high energy x-ray talbot-lau grating interferometer for industrial applications J. Korean Phys. Soc. 73 1827-33

[46] Josell D et al 2020 J. Electrochem. Soc. 167132504 